\title{
Molecular Excitation at the Nucleus of the QSO I Zw 1
}

\author{
A. Eckart \\ I.Physikalisches Institut, Universität zu Köln, Zülpicherstraße 77, 50937 \\ Köln, Germany \\ E. Schinnerer \\ Astronomy Department, California Institute of Technology, Pasadena, \\ $C A$ 91125, USA
}

J. Staguhn

University of Maryland, College Park, MD 20742-2421, USA

\begin{abstract}
We present results from the most recent Plateau de Bure observation of the ${ }^{12} \mathrm{CO}(2-1)$ line towards the nearby QSO I Zw 1.
\end{abstract}

With a redshift of $\mathrm{z}=0.0611 \mathrm{I} \mathrm{Zw} 1$ is one of the closest QSOs for which detailed interferometric studies of the host galaxy ISM are possible. I Zw 1 is interacting with a nearby companion east of the nuclear bulge at the edge of its optical disk and shows a number of properties also found in high redshift QSOs. The most recent observations of the ${ }^{12} \mathrm{CO}(2-1)$ line emission of $\mathrm{I} \mathrm{Zw} 1$ using the $\mathrm{PdBI}$ in its $\mathrm{B} / \mathrm{C}$ configuration have reached subarcsecond angular resolution (0.9" FWHM; Schinnerer et al. in prep.). Our recent ${ }^{12} \mathrm{CO}(1-0)$ data showed a $1.5 "(1.5 \mathrm{kpc})$ diameter ring-like distribution of the molecular gas and high resolution NIR observations indicate the presence of a circum-nuclear starburst (Schinnerer et al. 1998). In the pv-diagrams along the major kinematic axis at $\mathrm{PA}=135^{\circ}$ (Fig. 1 ) our new $\mathrm{J}=2-1$ emission line data is more centrally peaked compared to $\mathrm{J}=1-0$. This is consistent with our previous finding (Eckart et al. 1994) that the molecular gas in the disk of $\mathrm{I} \mathrm{Zw} 1$ is cold or sub-thermally excited (low line ratio $\left.\mathrm{R}=\mathrm{I}\left[{ }^{12} \mathrm{CO}(2-1)\right] / \mathrm{I}\left[{ }^{12} \mathrm{CO}(1-0)\right] \sim 0.5\right)$, whereas the emission from the nucleus is due to warm molecular gas with higher line rations. Fig.3 and Fig.4 demonstrate that the line emission from the ring itself is from warm optically thick molecular gas (ratio $\mathrm{R} \sim 0.9$ ) whereas the nuclear emission at smaller and larger radii is at least partly due to warm optically thin molecular gas $(R \geq 1.0)$ most likely heated by the nuclear star formation activity. The increase of the ${ }^{12} \mathrm{CO}(2-1)$ line intensity due to molecular excitation compensates the contrast that is required to get a sharper view of the ring-like structure seen in the ${ }^{12} \mathrm{CO}(1-0)$ line emission which is evident in subarcsecond interferometric ${ }^{12} \mathrm{CO}(1-0)$ data taken with BIMA (Staguhn, Schinnerer, Eckart in prep.).

\section{References}

Schinnerer et al. 1998, ApJ 500, 147

Eckart et al. 1994, ApJ 424, 627 


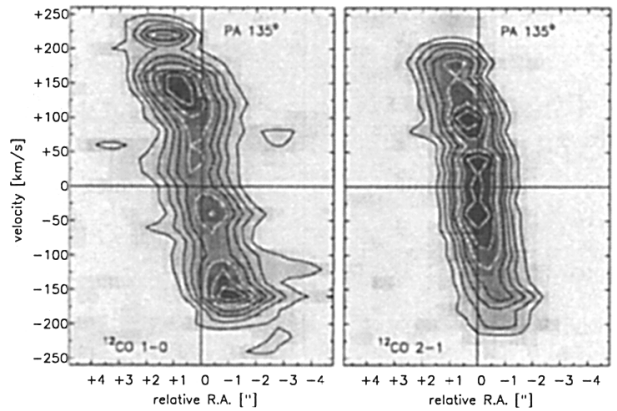

Figure 1. Position-velocity diagram (grey scale and contours) of the nuclear $12 \mathrm{CO}(1-0)$ (left) and the ${ }^{12} \mathrm{CO}(2-1)$ (right) line emission along the major kinematic axis. Contours are $30 \%, 40 \%, \ldots 100 \%$ of the peak flux of $0.034 \mathrm{Jy} /$ beam $(\mathrm{J}=1-0$ at $1.9 ")$ and $0.043 \mathrm{Jy} /$ beam ( $\mathrm{J}=2-1$ convolved to $1.9 ")$.

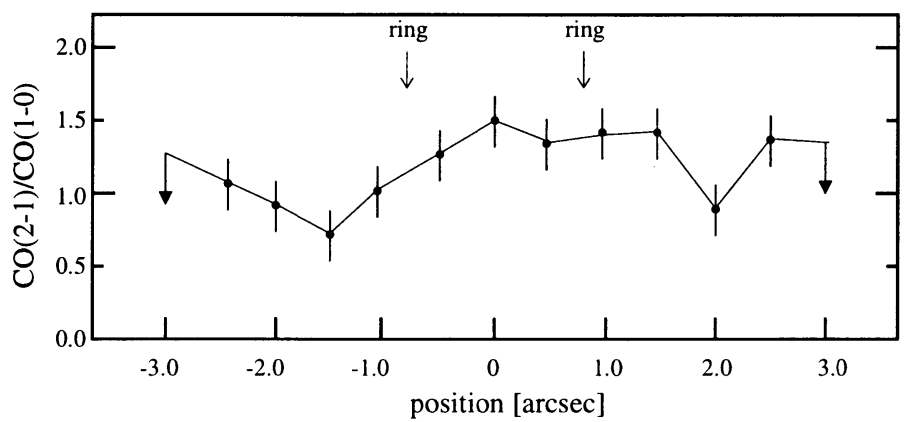

Figure 2. The ${ }^{12} \mathrm{CO}(2-1)$ over ${ }^{12} \mathrm{CO}(1-0)$ line intensity ratio as a function of position along the major kinematic axis at $\mathrm{PA}=135^{\circ}$ at an angular resolution of 1.9 "; 1 " corresponds to about $1 \mathrm{kpc}$.

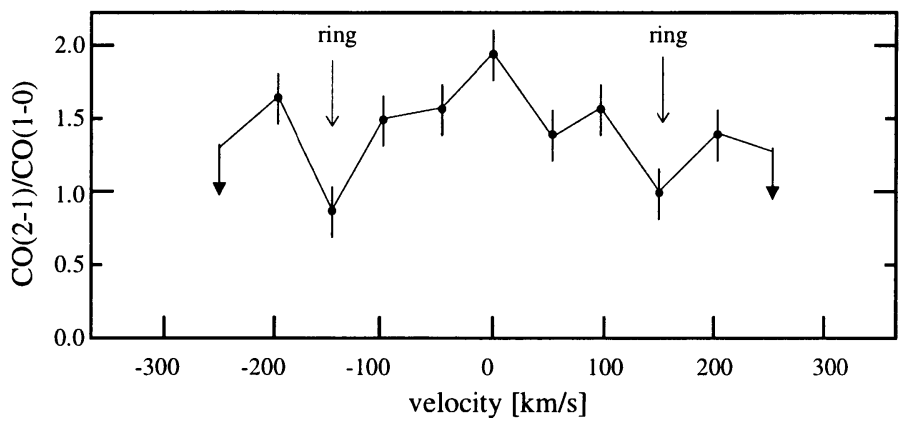

Figure 3. The ${ }^{12} \mathrm{CO}(2-1)$ over ${ }^{12} \mathrm{CO}(1-0)$ line intensity ratio as a function of velocity along the major kinematic axis at $\mathrm{PA}=135^{\circ}$. 